\title{
Time-Resolved Structural Dynamics of Organic Mixed Ionic Electronic Conductors
}

Bryan D. Paulsen ${ }^{1}$, Ruiheng Wu ${ }^{1}$, Christopher J. Takacs ${ }^{2}$, Hans-Georg Steinrück ${ }^{3}$, Joseph Strzalka ${ }^{4}$, Qingteng Zhang ${ }^{4}$, Michael F. Toney ${ }^{2}$, Jonathan Rivnay*1

1. Department of Biomedical Engineering, Northwestern University, Evanston, IL 60208, USA

2. Stanford Synchrotron Radiation Lightsource, SLAC National Accelerator Laboratory, Menlo Park, CA 94025, USA

3. Technische und Makromolekulare Chemie, Universität Paderborn, Warburger Str. 100, 33098 Paderborn, Germany

4. X-Ray Science Division, Argonne National Laboratory, Lemont, Illinois 60439, USA

*jrivnay@northwestern.edu

Keywords: operando, in situ, giwaxs, omiecs, pedot:pss

\section{Abstract}

The structure and packing of organic mixed ionic-electronic conductors have an outsized effect on transport properties. In operating devices this structure is not fixed but is responsive to changes in electrochemical potential, ion intercalation, and solvent swelling. Towards this end, the dynamic structure of a model organic mixed conductor is characterized using multimodal time-resolved operando techniques. Time-resolved operando X-ray scattering reveals asymmetric rates of structural change during doping and dedoping that do not directly depend on potential or charging dynamics. Time-resolved spectroscopy establishes a link between structural transients and the complex dynamics of electronic charge carrier subpopulations, in particular the polaron-bipolaron equilibrium. These findings provide insight into the factors limiting the response time of organic mixed conductor based devices, and present the first real-time observation of the structural changes during doping and dedoping of a conjugated polymer system via X-ray scattering. 
Organic mixed ionic-electronic conductors (OMIECs) are a class of conjugated materials ${ }^{[1]}$ of growing interest for bioelectronic, ${ }^{[2]}$ energy storage, ${ }^{[3]}$ electrochromic, ${ }^{[4]}$ and neuromorphic computing ${ }^{[5]}$ applications due to their ability to simultaneously transport both ionic and electronic charge, and couple between the two. Across applications, many OMIEC-based devices under operating conditions undergo changes in structure and system composition due to the uptake and expulsion of ions and solvent, and the modulation of electronic charge on the conjugated OMIEC backbone. ${ }^{[6-8]}$ This greatly complicates the establishment of predictive structure-property relationships with which to guide the design of next generation OMIECs, as static (often dry) structure is no longer sufficient for drawing these relationships. This has motivated the investigation of OMIECs and related materials with ex situ, in situ, and operando methods. Grazing incidence wide angle X-ray scattering (GIWAXS) has been widely employed to investigate conjugated materials, as the probed crystalline fraction, texture, and/or order generally dictate ultimate charge transport properties. ${ }^{[9,10]}$ Previously, GIWAXS has been carried out on ex situ electrochemically doped conjugated polymers ${ }^{[11]}$ and OMIECs ${ }^{[7]}$ to quantify their electrochemical potential dependent structural changes, generally manifesting as a $\pi$-stack spacing contraction and lamellar spacing expansion upon electrochemical doping. Ex situ methods intrinsically limit structural characterization to dry steady state conditions, and in the case of volatile solvents do not capture the contributions of solvent swelling. In situ and operando grazing incidence ${ }^{[12]}$ and transmission ${ }^{[13]} \mathrm{X}$-ray scattering methods have allowed for quantification of the electrochemical doping dependent steady state structure of conjugated polymers in contact with electrolytes under applied potentials. Nevertheless, thus far these methods could not be extended to time-resolved measurements to understand transient structural behavior far from steady state. For instance, the structure of a polythiophene in contact with a polymerized ionic liquid electrolyte measured with operando GIWAXS has been reported, ${ }^{[12]}$ however the very slow dynamics of the polymerized ionic liquid electrolyte prevent the investigation of any dynamic structural behavior of the conjugated polymer. For OMIEC applications the dynamic structure is of equal if not greater importance than the steady-state structure. 
Taking inspiration from the time-resolved GIWAXS investigation of conjugated polymers and OMIECS carried out during film formation and drying, ${ }^{[14,15]}$ we have carried out operando time-resolved GIWAXS of the prototypical OMIEC, PEDOT:PSS, during step potential transients, and in the more common steady-state potential regime. A variety of techniques have been employed to produce high electrical conductivity PEDOT:PSS films. ${ }^{[16]}$ One of the most effective methods to maximize electronic properties is crystallization via the exposure to sulfuric acid, ${ }^{[17]}$ which has been shown to produce high performance and high stability organic electrochemical transistors for bioelectronic applications. ${ }^{[18]}$ Acid crystallized PEDOT:PSS has the added benefit of producing reasonably ordered, well-textured films exhibiting high scattering intensities, making it an ideal candidate for in situ and operando GIWAXS measurements. Steady-state in situ structural characterization mapped the potential dependent scattering intensity and lattice spacings. Transient operando multi-modal structural and spectroscopic characterization revealed complex structural transients that unexpectedly diverged from simple charge or potential transients, but instead followed the polaron-bipolaron population equilibrium dynamics. These results have significant implications for the future design of OMIECs, especially in applications that require fast response times. 
a

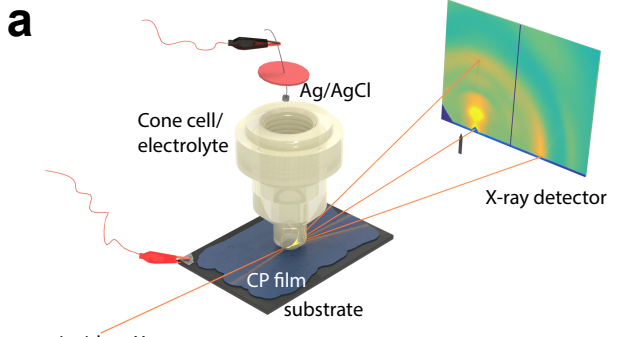

b

incident X-rays

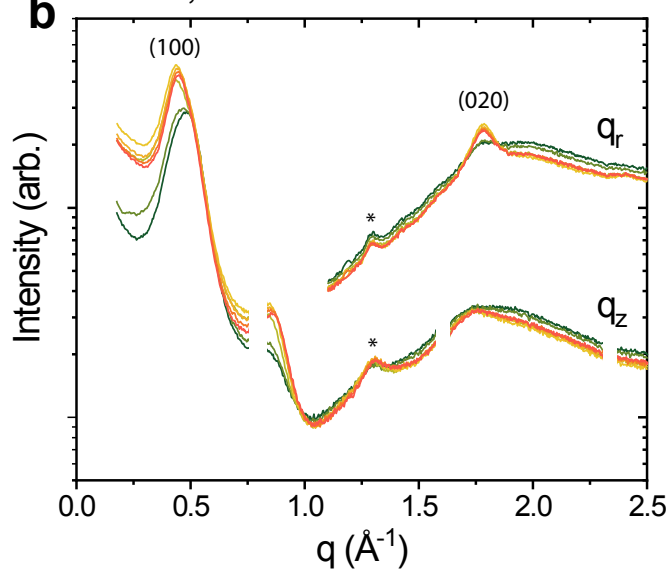

C

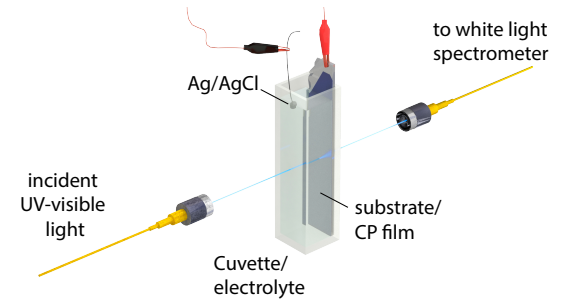

d

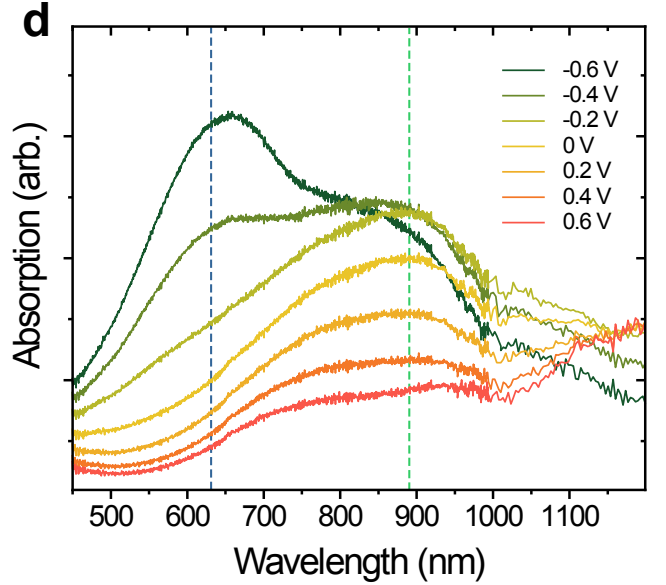

Figure 1. Operando scattering and spectroscopy of acid-crystallized PEDOT:PSS. Diagram of the (a) electrochemical cell with operando GIWAXS. (b) Nominally out-of-plane $\left(q_{z}\right)$ and in-plane $\left(q_{r}\right)$ line-cuts $\left(q_{r}>1.0\right.$ $\AA^{-1}$ ) taken from 2-D scattering plots of PEDOT:PSS films in the operando cell under applied potential (same legend as in (d)) highlighting the in-plane $\pi$-stack (020) and out-of-plane lamellar (100) scattering. Asterisks $\left({ }^{*}\right)$ denote parasitic scattering from PEEK cone cell. Diagram of the (c) UV-vis-NIR spectroelectrochemical cell. (d) UV-Vis-NIR spectra under various applied potentials highlighting the changes in the neutral $\pi-\pi^{*}$ and charged polaronic absorptions centered around $630 \mathrm{~nm}$ (blue dashed line) and $890 \mathrm{~nm}$ (green dashed line), respectively.

Static 2-D GIWAXS patterns of drop-cast acid crystallized PEDOT:PSS were measured (Figure S1a) revealing a structure consistent with previously reported literature. ${ }^{[17-20]}$ On the whole, the strong texturing and previously inferred high degree of crystallinity of acid crystallized PEDOT:PSS ${ }^{[17,18]}$ yield strong anisotropic scattering that makes it attractive for operando electrochemical experiments, allowing for shorter exposure times at the same incident flux, and the potential to overcome strong background scattering from the electrolyte and cell material.

The operando semicrystalline structure during electrochemical measurements was directly probed with GIWAXS using a machined polyether ether ketone (PEEK) cone cell that contacted the sample substrate defining the area of film exposed to electrolyte $\left(\sim 0.20 \mathrm{~cm}^{2}\right)$, Figure 1a. This cell is based on 
that developed for precision electrochemistry for electrochemical energy storage and is readily adopted for the present study. ${ }^{[21-24]}$ The machined PEEK wall thickness was sufficiently thin that the attenuation of the scattered intensity was not prohibitive (Figure S1b). Due to the machined knife edge, attenuation of the low angle incident beam was minimal, scattered intensity was increasingly attenuated with increasing scattering angle, reaching $\sim 50 \%$ attenuation at $25^{\circ}$, due to a PEEK pathlength of $\sim 1.1 \mathrm{~mm}$. The film thickness $(\sim 3-6 \mu \mathrm{m})$ and vertically focused beam height $(<10 \mu \mathrm{m})$ were optimized to minimize the electrolyte background scattering, allowing the resolution of the inplane $\pi$-stack and out-of-plane lamellar scattering from acid crystallized PEDOT:PSS immersed in aqueous electrolyte under applied electrochemical potential. 2-D GIWAXS patterns collected with the acid crystallized PEDOT:PSS inside the PEEK electrochemical cell filled with $100 \mathrm{mM}$ aqueous $\mathrm{NaCl}$ retain the edge-on oriented structure, and despite the large electrolyte scattering background, the inplane $\pi$-stack and out-of-plane lamellar scattering peaks are still resolvable (Figures $\mathbf{1 b}$ ).

In parallel to the operando GIWAXS measurements, electrochemistry with operando UV-vis-NIR transmission spectroscopy (spectroelectrochemistry) was carried out to probe the neutral and charged (polaron and bipolaron) states during electrochemical measurements (Figure 1c). Spectra from 400 to $1200 \mathrm{~nm}$ under applied electrochemical potential (Figure 1d) displayed a neutral $\pi-\pi^{*}$ absorption centered around $630 \mathrm{~nm}$, a polaronic absorption centered around $890 \mathrm{~nm}$, and the onset of a bipolaronic absorption beginning above $1000 \mathrm{~nm}$ and centered at a higher wavelength beyond the instrument range. ${ }^{[25-28]}$ These spectroelectrochemical measurements allowed the direct assessment of the relative neutral and polaron populations from the resolved neutral and polaron absorptions, and by inference the relative bipolaron population.

Electrochemically cycling the film (i.e. cyclic voltammetry) while simultaneously collecting 2-D GIWAXS patterns or UV-vis-NIR spectra allowed the dynamic tracking of the potential dependent lamellar and $\pi$-scattering behavior, along with relative neutral and polaron populations (Figure 2). The potential 
was swept at $5 \mathrm{mV} / \mathrm{s}$ to allow a longer X-ray detector integration time of $10 \mathrm{~s}$ for better peak resolution. The voltage and current density traces over time (Figure 2a,b) reflected a PEDOT:PSS film charging and discharging in a capacitive manner with relatively flat current plateaus. ${ }^{[29,30]}$ The potential range was limited to $\pm 0.6 \mathrm{~V}$ vs $\mathrm{Ag} / \mathrm{AgCl}$, as the applied voltages were low enough to avoid electrolyte breakdown yet large enough to probe the range of reproducible scattering peak shifts. Plotted as current density versus potential (Figure S2), the cyclic voltammograms display a capacitive hysteresis common to PEDOT:PSS films cycled over similar potential ranges. ${ }^{[31]}$ The out-of-plane lamellar scattering peak displayed the largest change during cycling, shifting to higher $q_{z}$ toward the negative extreme $(E<0 \mathrm{~V}$ vs $\mathrm{Ag} / \mathrm{AgCl}$ ) of the dedoping (reduction) sweep, returning to lower $q_{z}$ during initial doping (oxidation) sweep, reaching a minimum $q_{z}$ around $0 \mathrm{~V} v \mathrm{Ag} / \mathrm{AgCl}$ ), and relaxing to a slightly higher $q_{z}$ toward the positive extreme ( $\mathrm{E}>0 \mathrm{~V}$ vs $\mathrm{Ag} / \mathrm{AgCl})$ of the doping (oxidation) sweep, as shown in the line-cut colormap in Figure 2c. This pattern of $q_{z}$ lamellar peak shift proved to be reversible, repeating itself upon successive voltage sweeps (Figure S2b). Interestingly, the $\pi$-stack scattering does not show a significant shift in $q_{r}$ position from the strong underlying background, but instead was dramatically diminished in intensity upon reductive dedoping ( $\mathrm{E}>0 \mathrm{~V}$ vs $\mathrm{Ag} / \mathrm{AgCl}$ ), with a simultaneous increase in the scattering background at larger $q_{r}$, (Figure S3b). As with the lamellar peak shifts, the changes in the $\pi$-stack scattering proved to be reversible, repeating itself upon successive potential cycling.

The increased high- $q$ electrolyte scattering background during reductive dedoping $(\mathrm{E}>0 \mathrm{Vvs} \mathrm{Ag} / \mathrm{AgCl})$ was accompanied by a decrease in the low- $q$ scattering background and lamellar scattering intensity. The uptake of cations and water upon dedoping disrupted the packing of the PEDOT:PSS, broadening the (100) peak by about $35 \%$. After accounting for the experimental resolution, ${ }^{[32]}$ calculating the mean number of Bragg planes contributing to the scattering, $\overline{\mathrm{N}}_{100}=\mathrm{q}_{100} / \Delta \mathrm{q}_{100}$, showed a reduction from 3.9 to 3.0. Correspondingly, the intensity of the 100 peak, which is proportional to the square of the number of scatterers, ${ }^{[33]}$ for the dedoped state dropped to about $60 \%\left(3.0^{2} / 3.9^{2}\right)$ of the intensity observed for the doped state. In addition to the decrease in lamellar scattering, the low- $q$ scattering 
background at $-0.6 \mathrm{~V}$ vs $\mathrm{Ag} / \mathrm{AgCl}$ decreased $\sim 65 \%$ relative to the doped state. The changes in low- $q$ scattering background are ascribed to changes in film thickness, ${ }^{[34]}$ composition, and meso-scale structure that coincide with potential dependent swelling, and are the focus of future study.

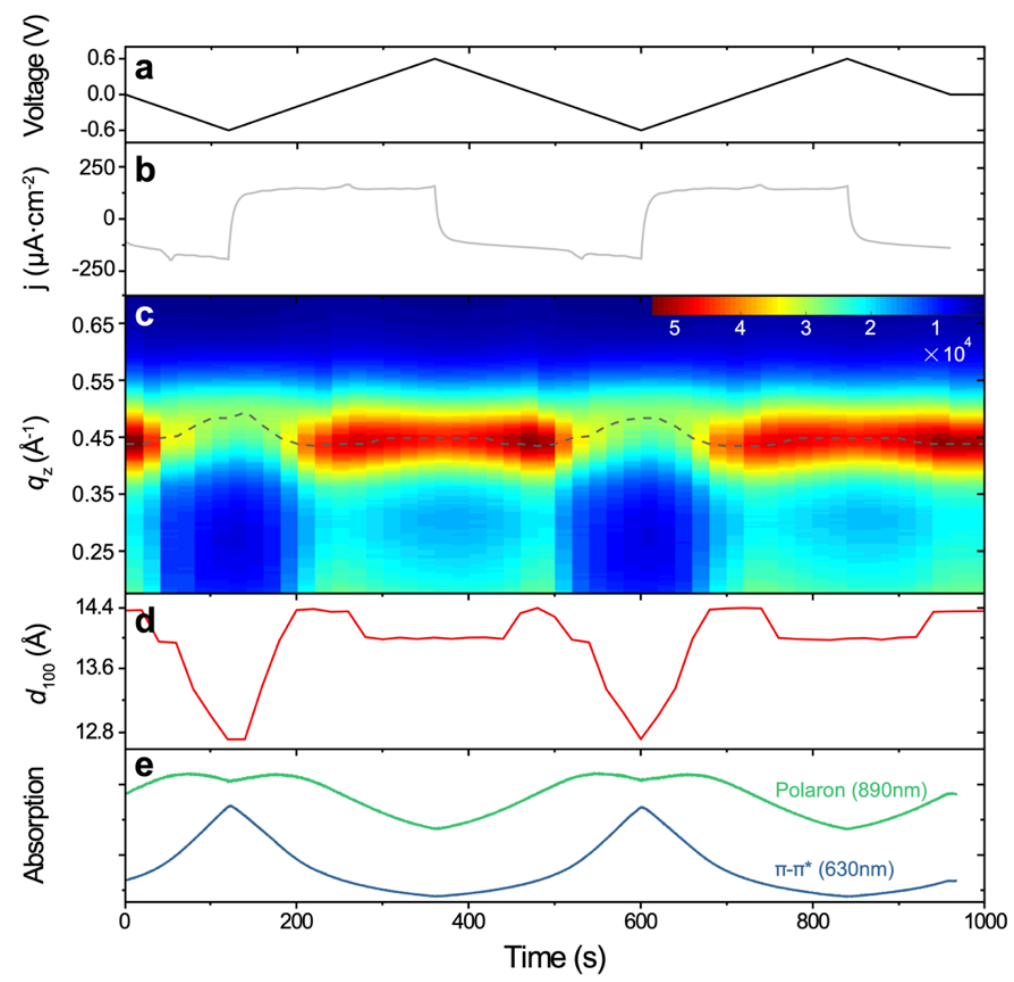

Figure 2. Cycled linear potential sweeps during operando GIWAXS and UV-vis-NIR spectroscopy: (a) Potential profile, (b) current density traces, (c) out-of-plane scattering intensity color map, (d) lamellar d-spacing, and (e) $630 \mathrm{~nm} \pi-\pi^{*}$ (blue line) and $890 \mathrm{~nm}$ polaronic (green line) absorption over time. The dotted line in (d) serves to indicate the maximum lamellar scattering intensity at each time step.

The position of the lamellar scattering was fit to calculate the average $d_{100}$ lamellar spacing plotted over time in Figure $\mathbf{2 d}$. The potential-dependent scattering behavior implies a contracted lamellar $\mathbf{d}$ spacing of $13.0 \AA \AA$ and relatively disordered $\pi$-stack in the (relatively) dedoped state $(\mathrm{E}<0 \mathrm{~V}$ vs $\mathrm{Ag} / \mathrm{AgCl})$. When doped $(\mathrm{E} \sim 0 \mathrm{~V}$ vs $\mathrm{Ag} / \mathrm{AgCl})$ the lamellar $\mathrm{d}$-spacing expands to $14.3 \AA$ and the $\pi$-stack becomes quite ordered with a d-spacing of $3.53 \AA$. This is roughly analogous to the often reported $\pi$-stack contraction and lamellar expansion seen in electrochemically or molecularly doped polyalkylthiophenes. ${ }^{[11-13,35,36]}$ Though in this case, any change in $\pi$-stack $d$-spacing was overshadowed by changes in $\pi$-stack scattering intensity. However, with further doping $(\mathrm{E}>0 \mathrm{~V} v \mathrm{Ag} / \mathrm{AgCl})$ while the 
$\pi$-stack maintains it degree of order and d-spacing, the lamellar d-spacing relaxes to $14.05 \AA$. Analogous cyclic spectroelectrochemical experiments revealed the polaron and $\pi-\pi^{*}$ absorption to roughly track the structural evolution (Figure $2 \mathrm{e}$ ), but with a local polaron minimum and $\pi-\pi^{*}$ maximum coinciding with the dedoped $d_{100}$ contraction minimum, and a global polaron minimum and $\pi-\pi^{*}$ minimum coinciding with the highly doped $d_{100}$ relaxation. The polaron absorption reached its maximum around $-0.2 \mathrm{Vvs} \mathrm{Ag} / \mathrm{AgCl}$, but was symmetric with potential sweep direction, unlike the $d_{100}$ which shows hysteretic behavior about $-0.2 \mathrm{~V}$ vs $\mathrm{Ag} / \mathrm{AgCl}$.

Whereas, in polyalkylthiophenes, further doping is likely to drive dopant anions into the sidechain lamellae, thus further expanding the lamellae spacing with increasing degree of doping, ${ }^{[8,11,12]}$ here the dopant anion is tethered to the PSS chains already present between PEDOT layers, thus increasing the degree of doping occurs by depleting the PSS chains of some of their charge balancing cations. Specifically, the further doping of PEDOT:PSS requires the loss of material (cations and their solvating water molecules) from between the PEDOT layers, resulting in a decrease in the lamellar $d$-spacing.

From comparing the dynamic potential cycled GIWAXS data with GIWAXS patterns collected after equilibrating at a fixed potential, the potential sweep rate $(5 \mathrm{mV} / \mathrm{s})$ was sufficiently slow as to closely approximate the equilibrated/steady-state potential dependent structure, Figure S3. The equilibrated constant potential GIWAXS data showed the same potential dependent $d$-spacing and scattering intensity behavior for $\mathrm{E}<0 \mathrm{~V}$ vs $\mathrm{Ag} / \mathrm{AgCl}$, confirming the $d_{100}$ hysteresis around $-0.2 \mathrm{~V} \mathrm{vs} \mathrm{Ag} / \mathrm{AgCl}$. However, for $\mathrm{E}>0 \mathrm{~V}$ vs $\mathrm{Ag} / \mathrm{AgCl}$, the equilibrated measurements showed the relaxed $d_{100}$ to have a slight potential dependence, with $d_{100}$ slightly decreasing (the (100) peak shifting to higher $q_{z}$, Figure S3a) with increasing positive voltage, indicating that this lamellar relaxation occurs over long time scales. 
From cyclic linear sweep spectroelectrochemistry, the neutral $\pi-\pi^{*}$ absorption centered about $630 \mathrm{~nm}$ was found to be strongest in the dedoped state under a reductive potential of $-0.6 \mathrm{~V} v \mathrm{Ag} / \mathrm{AgCl}$, and was successively bleached with application of more positive potential, (Figure 2e). This was to be expected as the high degree of doping and charge delocalization were expected to consume all the neutral species (thiophene rings) in the film. The polaron absorption centered about $630 \mathrm{~nm}$ was found to be strongest under potentials between -0.3 to $-0.4 \mathrm{~V} v \mathrm{Ag} / \mathrm{AgCl}$. At potentials more positive than $0.3 \mathrm{~V} v \mathrm{Ag} / \mathrm{AgCl}$ the polaron absorption diminished. This implies the increasingly preferential formation of bipolaronic charge species with further oxidative potential. Likewise, for potentials more negative than $-0.4 \mathrm{~V}$ vs $\mathrm{Ag} / \mathrm{AgCl}$ the polaron absorption diminished, as electronic charge was removed from the film and the polaronic species were converted back to neutral.

Under operating conditions, OMIECs present dynamic systems that are not necessarily at equilibrium. Therefore, time resolved GIWAXS was carried out during potential step and subsequent holding to track the transient structural evolution. Chronoamperometry was carried out by recording the current during potential steps to and from 0 and $\pm 0.6 \mathrm{~V}$ vs $\mathrm{Ag} / \mathrm{AgCl}$, during continuous $0.5 \mathrm{~s} \mathrm{GIWAXS}$ exposures (Figure $3 a \mathbf{a} \mathbf{b}$ ). The color map of the $q_{z}$ line cuts shows the evolution of the (100) peak position transients following potential steps (Figure 3c). Converting peak position to $d$-spacing, the largest change in $d_{100}(\sim 1.5 \AA$ ) occurred when stepping the potential to and from 0 and $-0.6 \mathrm{~V} \mathrm{vs} \mathrm{Ag} / \mathrm{AgCl}$, representing dedoping ( 0 to $-0.6 \mathrm{~V})$ and redoping $(-0.6$ to $0 \mathrm{~V}$ ) of the film (Figure $3 \mathrm{~d}$ ). Further modulation of the doping with potential steps to and from 0 and $+0.6 \mathrm{~V} v \mathrm{Ag} / \mathrm{AgCl}$ produced a moderate lamellar contraction from 14.3 to $14.05 \AA$.

The time scales of the lamellar $\mathrm{d}$-spacing changes accompanying the dedoping ( 0 to $-0.6 \mathrm{~V}$ ) and doping $(-0.6$ and $0 \mathrm{~V})$ steps were not symmetric, with the $d_{100}$ contraction during dedoping occurring faster than the $d_{100}$ expansion during doping. Empirically fitting the $d_{100}$ transients revealed the time constant during doping $\left(\tau_{d,-0.6 \rightarrow 0 v}=0.72 \pm 0.06 \mathrm{~s}\right)$ to be over twice that during dedoping $\left(\tau_{d, 0 \rightarrow 0.6 \mathrm{~V}}=0.32 \pm 0.17\right.$ 
s) (see equation S1). Moreover, during the slower process of doping the lamellar $d_{100}$ initially expanded to $14.4 \AA$, which was effectively (>95\%) complete in $4 \mathrm{~s}$. However, that represented an overshoot, which after $10 \mathrm{~s}$ began to slowly relax to its equilibrated value of $14.3 \AA$, (see from 50 to $60 \mathrm{~s}$ and from 130 to $140 \mathrm{~s}$ in Figure $3 \mathrm{~d}$ ). This is in contrast to the dedoping $d_{100}$ contraction to $12.9 \AA$, which was effectively (>95\%) complete in under $2 \mathrm{~s}$ and did not overshoot.
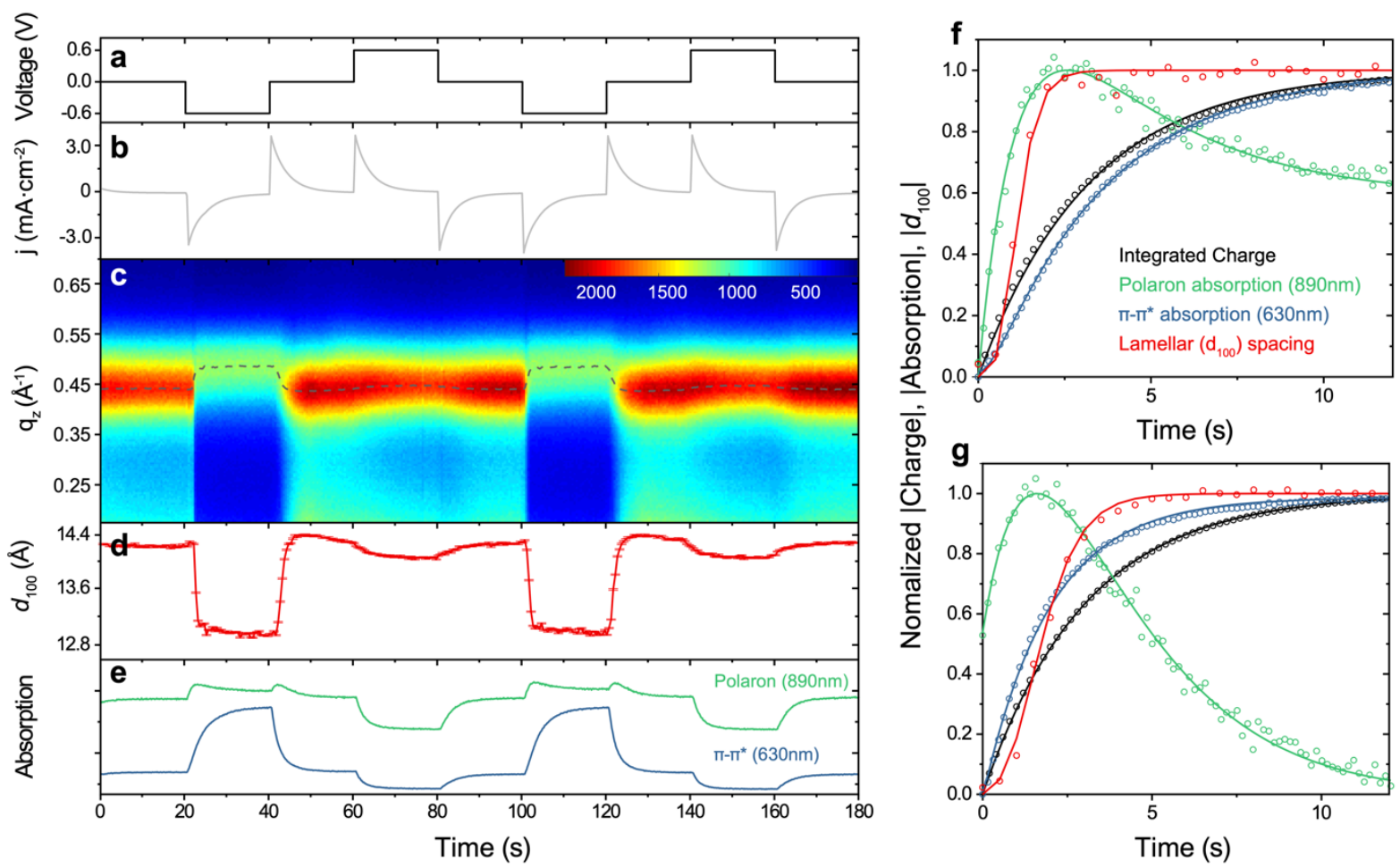

Figure 3. Potential step with time-resolved operando GIWAXS and UV-vis-NIR spectroscopy: (a) Potential profile, (b) charging current density traces, (c) out-of-plane scattering intensity color map, (d) lamellar dspacing, and (e) $630 \mathrm{~nm} \pi-\pi^{*}$ (blue line) and $890 \mathrm{~nm}$ polaronic (green line) absorption over time. The dotted line in (d) serves to indicate the maximum lamellar scattering intensity at each time step. Normalized changes in integrated charge, $890 \mathrm{~nm}$ polaronic absorption, $630 \mathrm{~nm} \pi-\pi^{*}$ absorption, and $d_{100}$ over time during (f) dedoping ( 0 to $-0.6 \mathrm{~V}$ vs $\mathrm{Ag} / \mathrm{AgCl}$ ) and (g) doping ( 0 to $-0.6 \mathrm{~V}$ vs $\mathrm{Ag} / \mathrm{AgCl}$ ) transients. For figure (f) and (g), open circles represent the experimental data and solid lines represent the model fits.

The forward and reverse potential steps from 0 and $+0.6 \mathrm{Vvs} \mathrm{Ag/AgCl}$ produced much slower and more symmetric $d_{100}$ transients, with both processes following roughly equivalent time constants $\left(\tau_{d, 0 \rightarrow 0.6 \mathrm{~V}}=\right.$ $\left.2.25 \pm 0.76 \mathrm{~s}, \tau_{d,+0.6 \rightarrow 0 \mathrm{~V}}=2.56 \pm 0.50 \mathrm{~s}\right)$ that were over seven times slower than the fast dedoping process. The overall transient behavior was not unique to the lamellar spacing but was mirrored by the 
transient behavior of the $\pi$-stack scattering intensity, and low and high $q$ scattering background, strongly implying that the potential dependent structural transformations occurs synchronously.

The simultaneously collected chronocoulometric data was readily fit with a simple RC model. The charging time constants for potentials steps between 0 and $-0.6 \mathrm{~V} \mathrm{vs} \mathrm{Ag} / \mathrm{AgCl}\left(\tau_{q,-0.6 \rightarrow 0 v}=3.69 \pm 0.74 \mathrm{~s}\right.$, $\tau_{q, 0 \rightarrow 0.6 V}=3.34 \pm 0.72 \mathrm{~s}$ ) (Figure $3 \mathrm{f}$ ) and between 0 and $+0.6 \mathrm{~V}$ vs $\mathrm{Ag} / \mathrm{AgCl}\left(\tau_{q,+0.6 \rightarrow 0 v}=3.58 \pm 0.54 \mathrm{~s}\right.$ $\left.\tau_{q, 0 \rightarrow+0.6 V}=3.34 \pm 0.72 \mathrm{~s}\right)$ where all quite similar. On the whole, the structural transients outpaced the electrochemical charging of the films (Table 1), showing that complete charging of the film was not the rate limiting process dictating the rate of structural change. Nor did the structural transients align with the expected potential transients in the PEDOT:PSS film, assuming RC behavior.

Table 1. Time and rate constants for charge, $d$-spacing, and absorption dynamics

\begin{tabular}{|c|c|c|c|c|c|c|c|}
\hline $\begin{array}{l}\Delta E[\mathrm{~V} \text { vs } \\
\mathrm{Ag} / \mathrm{AgCl}]\end{array}$ & $\tau_{q, \text { GIWAXS }}[\mathrm{s}]$ & $\tau_{d 100}[s]$ & $\tau_{q, U V \text {-vis }}[s]$ & $k_{n \rightarrow \mathrm{p}}\left[\mathrm{s}^{-1}\right]$ & $k_{p \rightarrow \mathrm{n}}\left[\mathrm{s}^{-1}\right]$ & $k_{p \rightarrow \mathrm{bp}}\left[\mathrm{s}^{-1}\right]$ & $k_{b p \rightarrow p}\left[\mathrm{~s}^{-1}\right]$ \\
\hline $0 \rightarrow-0.6$ & $3.34 \pm 0.72$ & $0.32 \pm 0.17$ & $3.35 \pm 0.40$ & -- & $0.26 \pm 0.08^{b)}$ & -- & $0.93 \pm 0.15^{c}$ \\
\hline$-0.6 \rightarrow 0$ & $3.69 \pm 0.74$ & $0.72 \pm 0.06$ & $3.00 \pm 0.01$ & $0.50 \pm 0.01^{a)}$ & -- & $0.42 \pm 0.02^{c)}$ & -- \\
\hline $0 \rightarrow+0.6$ & $3.34 \pm 0.72$ & $2.25 \pm 0.76$ & $3.00 \pm 0.22$ & $\left.0.54 \pm 0.04^{a}\right)$ & -- & $\left.0.48 \pm 0.01^{c}\right)$ & -- \\
\hline$+0.6 \rightarrow 0$ & $3.58 \pm 0.54$ & $2.56 \pm 0.50$ & $2.58 \pm 0.03$ & -- & $0.29 \pm 0.01^{a)}$ & -- & $0.34 \pm 0.01^{c)}$ \\
\hline
\end{tabular}

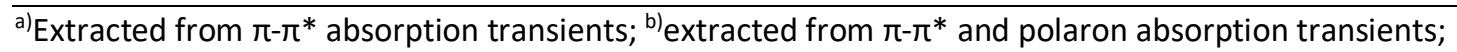

c) extracted from polaron absorption transients. $95 \%$ confidence intervals were calculated for the fit constants. Time and rate constants listed were averaged across equivalent potential steps. The error listed above is the larger of either the fit 95\% confidence interval of the fit parameters or two standard deviations of the averaged distribution. The asymmetric structural time constants and their related limiting kinetic constants are in bold.

In order to further probe this lack of correlation between structural and electrochemical transients, analogous transient spectroelectrochemical experiments were carried out. Operando transmission UV-vis-NIR spectra were collected during transient potential steps (Figure 3e). While the optical absorption data correlated with total charge, it also gave insight into the relative populations of neutral states, singly charged polarons, and dually charged bipolarons on the PEDOT backbone.

The absorption dynamics were modelled as two consecutive elementary reactions: 
$\underset{k_{p \rightarrow n}}{\stackrel{k_{n \rightarrow p}}{\rightleftharpoons} p+e^{-} \stackrel{k_{p \rightarrow b p}}{\rightleftharpoons} b p+e^{-}} \underset{k_{b p \rightarrow p}}{\rightleftharpoons} b$

where equilibrium population of neutral $(n)$, polaronic $(p)$, and bipolaronic $(b p)$ species was determined by the electrochemical potential, and the polaron population was an intermediary between the neutral and bipolaronic species. The concentrations of $n$ and $p$ were directly proportional to the 630 and $890 \mathrm{~nm}$ absorptions, respectively, and from their transient response the forward and reverse rate constants for both elementary reactions were calculated (see Equation S3 through S7).

The neutral-polaron kinetic parameters extracted from both $\pi-\pi^{*}$ and polaronic absorption transients were consistent across all potential step measurements, with the average neutral-to-polaron rate constant $\left(k_{n \rightarrow p}=0.521 \pm 0.047 \mathrm{~s}^{-1}\right)$ being twice the average of the reverse polaron-to-neutral rate constant $\left(k_{p \rightarrow n}=0.269 \pm 0.066 \mathrm{~s}^{-1}\right)$. Likewise, the polaron-to-bipolaron rate constants as determined from the polaron bleach dynamics were consistent giving an average value of $k_{p \rightarrow b p}=0.451 \pm 0.075 \mathrm{~s}$

${ }^{1}$. However, the extracted bipolaron-to-polaron rate constants varied significantly. During the 0 and $0.6 \mathrm{~V}$ vs $\mathrm{Ag} / \mathrm{AgCl}$ dedoping potential step, the bipolaron-to-polaron dynamics were fast, with $k_{b p \rightarrow p}=$ $0.93 \pm 0.15 \mathrm{~s}^{-1}$ and $>1 \mathrm{~s}^{-1}$ as determined from the bleach of the polaron absorption and the growth of the neutral absorption, respectively. Conversely, modulating the degree of doping by stepping the potential from +0.6 and $0 \mathrm{~V}$ vs $\mathrm{Ag} / \mathrm{AgCl}$, resulted in much slower bipolaron-to-polaron conversion, with an extracted rate constant of only $k_{b p \rightarrow p}=0.337 \pm 0.005 \mathrm{~s}^{-1}$.

It is possible that the structural dynamics would follow one or both of the charged population dynamics ( $p$ and/or bp). However, the polaron-to-neutral dynamics does not clarify the structural dynamics, as the asymmetric rapid lamellar contraction during dedoping ( 0 to $-0.6 \mathrm{~V} \mathrm{vs} \mathrm{Ag} / \mathrm{AgCl}$ ) and slower expansion during doping $(-0.6$ to $0 \mathrm{~V} \mathrm{vs} \mathrm{Ag} / \mathrm{AgCl})$ is the inverse of the slower polaron-to-neutral kinetics during dedoping and faster neutral-to-polaron kinetics during doping (Figure 3f,g). Nor can the lamellar spacing depend on the total polaron concentration, as shown above (Figure 2d,e), the polaron 
absorption does not show any hysteresis about $-0.2 \mathrm{~V} \mathrm{vs} \mathrm{Ag} / \mathrm{AgCl}$, whereas the lamellar spacing does (Figure S2b). Further, the initial transient increases in polaron concentration upon both 0 to -0.6 and 0.6 to $0 \mathrm{~V} \mathrm{vs} \mathrm{Ag/AgCl}$ potential steps (Figure 3e) should both be (but are not) accompanied by similar $d_{100}$ contractions (Figure $3 d$ ). Instead, the asymmetric structural dynamics correlate with the variable bipolaron-to-polaron rates.

The rapid depletion of bipolaronic species and production of polaronic species during dedoping (0 to $0.6 \mathrm{~V}$ vs $\mathrm{Ag} / \mathrm{AgCl}$ ) is followed in quick succession by a similarly rapid $d_{100}$ contraction, as seen in the initial rise in polaronic absorption and change in $d_{100}$ (Figure 3f). By the time polaronic absorption begins to fall, due to the rate of conversion of polaronic species to neutral species overtakes the rate of production of polaronic species from bipolaronic species, the $d_{100}$ has equilibrated. Both the $\pi-\pi^{*}$ absorption and the total integrated charge lag considerably behind the $d_{100}$ change. Conversely during doping (-0.6 to $0 \mathrm{~V}$ vs $\mathrm{Ag} / \mathrm{AgCl}), d_{100}$ is slow change until the polaronic absorption begins to fall, this time due to the rate of conversion of polaronic species from neutral species being overtaken by the rate of production of polaronic species from bipolaronic species, at which point $d_{100}$ then equilibrates (Figure 3g). Again, both the $\pi-\pi^{*}$ absorption and the total integrated charge lag considerably behind the $d_{100}$ change. In both cases the $d_{100}$ change clearly follows the dynamics of conversion between polaronic and bipolaronic species. Likewise, the slower $d_{100}$ expansion when stepping from - 0.6 to $0 \mathrm{~V}$ vs $\mathrm{Ag} / \mathrm{AgCl}$ and relaxation when stepping between 0 and $+0.6 \mathrm{~V}$ vs $\mathrm{Ag} / \mathrm{AgCl}$ seems to follow the slower polaron-bipolaron kinetics during those potential steps.

While the polaron-bipolaron kinetics dictate the $d_{100}$ dynamics, the physical explanation for the forward and reverse difference of these kinetics is not directly obvious. Often asymmetric or hysteretic behavior is attributed to electronic charge trapping. If significant electronic charge were trapped in the film, one would expect a delay in the extraction of charge during dedoping potential steps. However, here the charge extraction and injection during dedoping and doping potential steps was essentially 
identical (Figure $3 \mathrm{f}-\mathrm{g}$ and Table 1), making it unlikely that charge trapping was the source of the asymmetric structural dynamics and polaron-bipolaron kinetics.

Our simple kinetic model presumes that the only reaction pathway between polaronic and bipolaronic species is the addition or removal of an electron. It is also possible that two polarons can combine to form a bipolaron, or the reverse, a bipolaron may split into two polarons.

$$
p+p \underset{k_{b p \rightarrow p}^{\prime}}{\stackrel{k_{p \rightarrow b p}^{\prime}}{\rightleftharpoons}} b p
$$

This reaction pathway may play a significant role when the system is stepped far from its doped equilibrium during the 0 to $-0.6 \mathrm{~V} v \mathrm{Ag} / \mathrm{AgCl}$ dedoping step, thus leading to the perceived increase in the bipolaron-to-polaron rate constant.

While the admittedly simplistic kinetic model accounts for the absorption transients observed here, the potential dependent charge carrier populations are likely more complicated, including multi polaronic and bipolaronic species in addition to simple polarons and bipolarons. ${ }^{[28]}$ While this complicates the charge carrier classification, the absorption dynamics remain the same, with the dynamics of the absorption centered around $890 \mathrm{~nm}$ still seemingly linked to the $d_{100}$ dynamics.

In conclusion, we have for the first time measured the time resolved structural evolution of a conjugated polymer during electrochemical doping and dedoping via operando GIWAXS. Compared to the steady-state in situ measurements, the time-resolved operando GIWAXS revealed asymmetric rates of structural change. Operando spectroscopy revealed that the transient structural behavior is tied to the complex transient polaron-bipolaron dynamics. This is an interesting result, as much of the work to improve OMIEC device time response has focused on improving ion transport ${ }^{[37]}$ and reducing device dimensions. ${ }^{[38,39]}$ This work shows that even in such optimized settings bipolaron population 
dynamics likely present a limit to device speed. This begs the question, can OMIECs be designed to tailor electronic charge carrier population dynamics? As of now, this is an unexplored area of research.

\section{Acknowledgements}

B.P., R.W., and J.R. gratefully acknowledge support from the National Science Foundation Grant No. NSF DMR-1751308. This research used resources of the Advanced Photon Source, a U.S. Department of Energy (DOE) Office of Science User Facility operated for the DOE Office of Science by Argonne National Laboratory under Contract No. DE-AC02-06CH11357. Use of the Stanford Synchrotron Radiation Lightsource, SLAC National Accelerator Laboratory, is supported by the U.S. Department of Energy, Office of Science, Office of Basic Energy Sciences under Contract No. DE-AC02-76SF00515. Special thanks to J. Busan and the Northwestern University Research Machine Shop. 


\section{References}

[1] B. D. Paulsen, K. Tybrandt, E. Stavrinidou, J. Rivnay, Nature Materials 2020, 19, 13.

[2] J. Rivnay, R. M. Owens, G. G. Malliaras, Chem. Mater. 2014, 26, 679.

[3] S. Muench, A. Wild, C. Friebe, B. Häupler, T. Janoschka, U. S. Schubert, Chem. Rev. 2016, 116, 9438.

[4] P. M. Beaujuge, J. R. Reynolds, Chem. Rev. 2010, 110, 268.

[5] Y. van de Burgt, A. Melianas, S. T. Keene, G. Malliaras, A. Salleo, Nature Electronics 2018, 1, 386.

[6] L. Q. Flagg, R. Giridharagopal, J. Guo, D. S. Ginger, Chem. Mater. 2018, $30,5380$.

[7] C. Cendra, A. Giovannitti, A. Savva, V. Venkatraman, I. McCulloch, A. Salleo, S. Inal, J. Rivnay, Advanced Functional Materials 2019, 29, 1807034.

[8] C. G. Bischak, L. Q. Flagg, K. Yan, T. Rehman, D. W. Davies, R. J. Quezada, J. W. Onorato, C. K. Luscombe, Y. Diao, C.-Z. Li, D. S. Ginger, arXiv:1910.06440 [cond-mat, physics:physics] 2019.

[9] R. Noriega, J. Rivnay, K. Vandewal, F. P. V. Koch, N. Stingelin, P. Smith, M. F. Toney, A. Salleo, Nature Materials 2013, 12, 1038.

[10] S. Fratini, M. Nikolka, A. Salleo, G. Schweicher, H. Sirringhaus, Nature Materials 2020, 19, 491.

[11] J. O. Guardado, A. Salleo, Advanced Functional Materials 2017, 27, DOI 10.1002/adfm.201701791.

[12] E. M. Thomas, M. A. Brady, H. Nakayama, B. C. Popere, R. A. Segalman, M. L. Chabinyc, Advanced Functional Materials 2018, 28, 1803687.

[13] J. L. Thelen, S.-L. Wu, A. E. Javier, V. Srinivasan, N. P. Balsara, S. N. Patel, ACS Macro Lett. 2015, 4, 1386.

[14] C. M. Palumbiny, F. Liu, T. P. Russell, A. Hexemer, C. Wang, P. Müller-Buschbaum, Advanced Materials 2015, 27, 3391.

[15] E. F. Manley, J. Strzalka, T. J. Fauvell, N. E. Jackson, M. J. Leonardi, N. D. Eastham, T. J. Marks, L. X. Chen, Advanced Materials 2017, 29, 1703933.

[16] H. Shi, C. Liu, Q. Jiang, J. Xu, Advanced Electronic Materials 2015, 1, 1500017.

[17] N. Kim, S. Kee, S. H. Lee, B. H. Lee, Y. H. Kahng, Y.-R. Jo, B.-J. Kim, K. Lee, Advanced Materials 2014, 26, 2268.

[18] S.-M. Kim, C.-H. Kim, Y. Kim, N. Kim, W.-J. Lee, E.-H. Lee, D. Kim, S. Park, K. Lee, J. Rivnay, M.-H. Yoon, Nature Communications 2018, 9, 3858.

[19] J. Rivnay, S. Inal, B. A. Collins, M. Sessolo, E. Stavrinidou, X. Strakosas, C. Tassone, D. M. Delongchamp, G. G. Malliaras, Nature Communications 2016, 7, 11287.

[20] Y. Honma, K. Itoh, H. Masunaga, A. Fujiwara, T. Nishizaki, S. Iguchi, T. Sasaki, Advanced Electronic Materials 2018, 4, 1700490.

[21] Y. W. Chen, J. D. Prange, S. Dühnen, Y. Park, M. Gunji, C. E. D. Chidsey, P. C. Mclntyre, Nature Materials 2011, 10, 539.

[22] P. F. Satterthwaite, A. G. Scheuermann, P. K. Hurley, C. E. D. Chidsey, P. C. McIntyre, ACS Appl. Mater. Interfaces 2016, 8, 13140.

[23] C. Cao, I. I. Abate, E. Sivonxay, B. Shyam, C. Jia, B. Moritz, T. P. Devereaux, K. A. Persson, H.-G. Steinrück, M. F. Toney, Joule 2019, 3, 762.

[24] H.-G. Steinrück, C. Cao, G. M. Veith, M. F. Toney, J. Chem. Phys. 2020, 152, 084702.

[25] O. Bubnova, Z. U. Khan, A. Malti, S. Braun, M. Fahlman, M. Berggren, X. Crispin, Nat Mater 2011, 10, 429.

[26] N. Massonnet, A. Carella, O. Jaudouin, P. Rannou, G. Laval, C. Celle, J.-P. Simonato, Journal of Materials Chemistry C 2014, 2, 1278.

[27] S. Rudd, J. F. Franco-Gonzalez, S. K. Singh, Z. U. Khan, X. Crispin, J. W. Andreasen, I. Zozoulenko, D. Evans, Journal of Polymer Science Part B: Polymer Physics 2018, 56, 97.

[28] I. Zozoulenko, A. Singh, S. K. Singh, V. Gueskine, X. Crispin, M. Berggren, ACS Appl. Polym. Mater. 2019, 1, 83.

[29] A. V. Volkov, K. Wijeratne, E. Mitraka, U. Ail, D. Zhao, K. Tybrandt, J. W. Andreasen, M. Berggren, X. Crispin, I. V. Zozoulenko, Advanced Functional Materials 2017, 27, 1700329. 
[30] K. Tybrandt, I. V. Zozoulenko, M. Berggren, Science Advances 2017, 3, eaao3659.

[31] A. Giovannitti, R. B. Rashid, Q. Thiburce, B. D. Paulsen, C. Cendra, K. Thorley, D. Moia, J. T. Mefford, D. Hanifi, D. Weiyuan, M. Moser, A. Salleo, J. Nelson, I. McCulloch, J. Rivnay, Adv. Mater. 2020, 1908047.

[32] D.-M. Smilgies, J Appl Cryst 2009, 42, 1030.

[33] B. E. Warren, X-Ray Diffraction, Dover, New York, 1990.

[34] A. Savva, S. Wustoni, S. Inal, J. Mater. Chem. C 2018, 6, 12023.

[35] J. E. Cochran, M. J. N. Junk, A. M. Glaudell, P. L. Miller, J. S. Cowart, M. F. Toney, C. J. Hawker, B. F. Chmelka, M. L. Chabinyc, Macromolecules 2014, 47, 6836.

[36] D. T. Duong, C. Wang, E. Antono, M. F. Toney, A. Salleo, Organic Electronics 2013, 14, 1330.

[37] G. D. Spyropoulos, J. N. Gelinas, D. Khodagholy, Science Advances 2019, 5, eaau7378.

[38] J. Rivnay, P. Leleux, M. Ferro, M. Sessolo, A. Williamson, D. A. Koutsouras, D. Khodagholy, M. Ramuz, X. Strakosas, R. M. Owens, C. Benar, J.-M. Badier, C. Bernard, G. G. Malliaras, Science Advances 2015, 1, e1400251.

[39] P. D’Angelo, S. L. Marasso, A. Verna, A. Ballesio, M. Parmeggiani, A. Sanginario, G. Tarabella, D. Demarchi, C. F. Pirri, M. Cocuzza, S. lannotta, Small 2019, 15, 1902332.

[40] Z. Jiang, Journal of Applied Crystallography 2015, 48, 917.

[41] K. E. Aasmundtveit, E. J. Samuelsen, L. A. A. Pettersson, O. Inganäs, T. Johansson, R. Feidenhans'l, Synthetic Metals 1999, 101, 561.

[42] K. E. Aasmundtveit, E. J. Samuelsen, O. Inganäs, L. A. A. Pettersson, T. Johansson, S. Ferrer, Synthetic Metals 2000, 113, 93.

[43] E.-G. Kim, J.-L. Brédas, J. Am. Chem. Soc. 2008, 130, 16880.

[44] M. N. Gueye, A. Carella, N. Massonnet, E. Yvenou, S. Brenet, J. Faure-Vincent, S. Pouget, F. Rieutord, H. Okuno, A. Benayad, R. Demadrille, J.-P. Simonato, Chem. Mater. 2016, 28, 3462. 


\section{Supporting Information}

\section{Time-Resolved Structural Dynamics of Organic Mixed Ionic Electronic Conductors}

Bryan D. Paulsen ${ }^{1}$, Ruiheng $\mathrm{Wu}^{1}$, Christopher J. Takacs ${ }^{2}$, Hans-Georg Steinrück ${ }^{3}$, Joseph Strzalka ${ }^{4}$, Qingteng Zhang ${ }^{4}$, Michael F. Toney ${ }^{2}$, Jonathan Rivnay*1

\section{Experimental Section}

Materials and Sample Preparation:

PEDOT:PSS (Clevios PH-1000), purchased from Heraeus Holding GmbH, was filtered through $0.45 \mu \mathrm{m}$ PES filters to remove large particles. For spectroelectrochemistry samples, the filtrate was spin-coated for $600 \mathrm{rpm}$ for $60 \mathrm{~s}$ on pieces of $1 \times 3 \mathrm{~cm}^{2}$ ITO coated glasses $\left(15-20 \mathrm{ohm} \mathrm{sq}{ }^{-1}\right.$ ) (University Wafer) that prior to spinning had been successively sonicated in acetone and isopropyl alcohol, and cleaned with UV-ozone exposure. The cast films were dried on a hotplate in ambient at $120{ }^{\circ} \mathrm{C}$ for 10 minutes to remove water and then immersed into concentrated $\mathrm{H}_{2} \mathrm{SO}_{4}$ (Sigma-Aldrich, 95.0\% - 98.0\%) for 10 minutes at room temperature. After which the films were rinsed with DI water 3 times. and dried on a hotplate in ambient at $120^{\circ} \mathrm{C}$ for 10 minutes. For the X-Ray scattering samples, the filtrate was dropcasted on a $1.5 \times 3.5 \mathrm{~cm}^{2}$ pieces of polished degenerately p-doped Si wafer $\left(0.001-0.005 \mathrm{ohm} \mathrm{cm}^{-1}\right)$ (University Wafer) at $70{ }^{\circ} \mathrm{C}$ that prior to drop-casting had been successively sonicated in acetone and isopropyl alcohol, and cleaned with UV-ozone exposure. The drop-cast films were dried by the same method described above. Due to the thickness of the drop-cast film, the acid treat time was prolonged to 3 hours. After rinsing, the sample was first heated at $60{ }^{\circ} \mathrm{C}$ for 60 minutes and then $120{ }^{\circ} \mathrm{C}$ for 30 minutes on a hotplate in ambient.

Operando Visible-NIR Spectroscopy:

Spectroelectrochemistry of acid crystallized PEDOT:PSS coated on ITO glass electrode was carried out in $100 \mathrm{mM}$ aqueous $\mathrm{NaCl}$ in a PMMA cuvette with a $\mathrm{Ag} / \mathrm{AgCl}$ pellet (Warner Instruments) reference/counter electrode. Potential control and current measurement was carried out with a 
potentiostat (Ivium). Simultaneous absorption spectroscopy was carried out with a halogen white light source (Ocean Optics, DH-2000-BAL) and an optical fiber light path split to separate UV-visible (Ocean Optics, FLAME-S) and near-infrared (Ocean Optics, NQ512) spectrometers, with 30 ms and 200 ms, resepctively. Electrochemical and spectroscopic data was recorded with Iviumsoft and OceanView software respectively. All analysis including curve merging, line cutting, and curve fitting were executed with MATLAB software.

\section{Operando Grazing-Incidence Wide-Angle X-Ray Scattering:}

All time and voltage dependent GIWAXS measurements were performed at beamline 8-ID-E of the Advanced Photon Source, Argonne National Laboratory with $10.92 \mathrm{keV}$ synchrotron radiation, $200 \mu \mathrm{m}$ wide, focused to a $10 \mu \mathrm{m}$ height, impinging on the sample with an incident angle of $0.14^{\circ}$ (selected to be between the critical angle of the polymer and $\mathrm{Si}$ substrate, $\sim 0.12^{\circ}$ and $0.164^{\circ}$ respectively), producing a $4 \mathrm{~mm}$ long beam foot print. The beam was vertically focused at the sample by a Be compound refractive lens composed of 17 lenslets with radius $0.2 \mathrm{~mm}$ positioned $2.175 \mathrm{~m}$ upstream of the sample The scattering was carried out under He atmosphere at room temperature with images collected by a Pilatus 1MF pixel array detector $228.2 \mathrm{~mm}$ away from the sample.

The operando cell was formed by pressing a machined knife edge polyether ether ketone (PEEK) cone against the PEDOT:PSS sample on a Si substrate supported by a polished quartz disk in a custom lens tube (Thorlabs) assembly. Electrochemical measurement and control employed the same potentiostat and reference/counter electrode employed in the above described spectroelectrochemistry. The operando GIWAXS cells were sealed with a PTFE coated silicone septum. For the CV and equilibrated step measurements, the integration time was set with $10 \mathrm{~s}$ to achieve a high signal to noise ratio. While the integration time for transient measurement was set to $0.5 \mathrm{~s}$ to capture structural dynamics. Good fits of the transient lamellar peaks required the combination of a Voigt peak with a varying center, width, and area, and a gaussian peak with varying width and area, but center fixed at $q_{z}=0.51 \AA^{-1}$. The 
transient $d_{100}$ values were calculated from the $q_{z}$ value at the maximum intensity of the summation of these two fit peaks, which represented the average $d_{100}$ at each given time step. All the GIWAXS data collected were processed with the GIXSGUI package for MATLAB. ${ }^{[40]}$

\section{Static GIWAXS.}

The 2-D scattering data (Figure S1) revealed a highly textured edge-on oriented structure, strong outof-plane lamellar scattering (hOO) and strong in-plane $\pi$-stack scattering $(0 / 0)$. In-plane $\left(q_{r}\right)$ and out-ofplane $\left(q_{z}\right)$ line cuts highlight the absence of lamellar ordering and the preponderance of $\pi$-stacking (020) in the plane of the film, and a strong lamellar scattering peak (100) and second order shoulder (200) out of the plane of the film. An in-plane peak at higher scattering vector $\left(q_{r} \sim 2.5 \AA^{-1}\right)$ is consistent with the PSS backbone scattering, ${ }^{[20]}$ while the out-of-plane feature at $\left(q_{z} \sim 1.35 \AA^{-1}\right)$ appears to be a conflation of the PSS chain stacking ${ }^{[14]}$ and the third order lamellar scattering. Some out-of-plane $\pi-\pi$ stacking persists $\left(q_{z} \sim 1.8 \AA^{-1}\right)$, consistent with previous structural studies of acid crystalized PEDOT:PSS. ${ }^{[18]}$ These data were consistent with the generally held crystal structure of lamellae of $\pi-$ stacked PEDOT chains, separated by a layer of compensating anions (Tosylate or PSS chains). ${ }^{[14,17-20,41-}$ ${ }^{44]}$ The calculated $\pi$-stack and lamellar spacings of $3.54 \AA$ and $13.3 \AA$, respectively, are in good agreement with the d-spacings of PEDOT:PSS and in particular with the previous reports of acid crystallized PEDOT:PSS. 
a

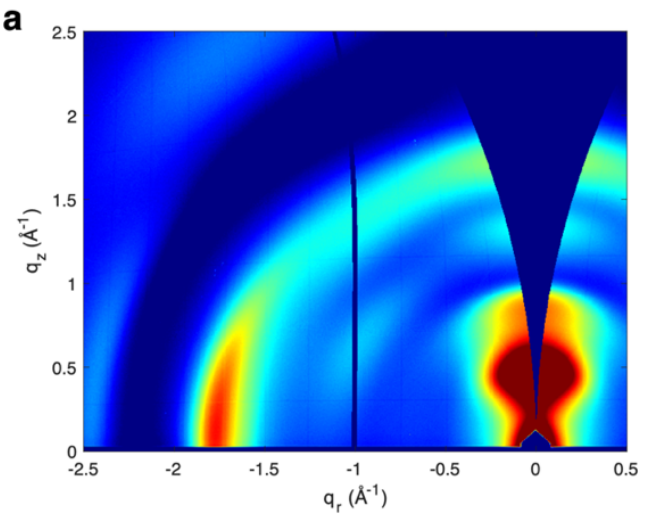

b

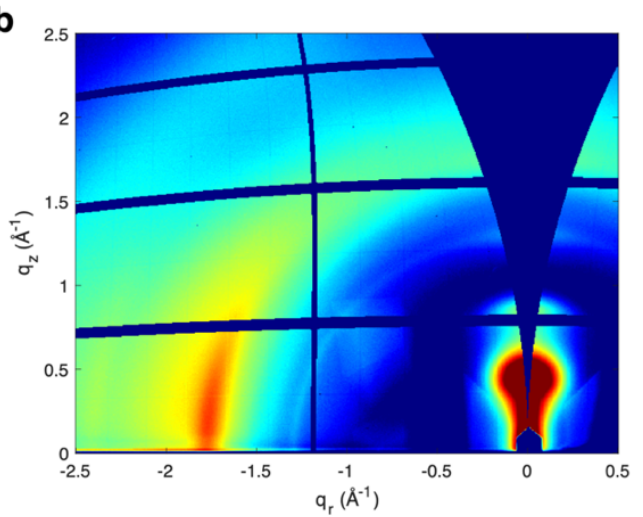

Figure S1. Two-dimensional grazing incidence X-ray $q_{r}-q_{z}$ scattering map of acid crystallized PEDOT:PSS (a) as cast and (b) in the operando cone cell filled with aqueous $100 \mathrm{mM} \mathrm{NaCl}$ electrolyte. The static $q_{r}-q_{z}$ scattering map (a) was stitched together from images collected with different vertical detector position to fill in the gaps between detector pixel array modules. Note in (b) that despite the increased diffuse (electrolyte) scattering at high $q$ and the shadowing from the operando cell at low $q$, the out-of-plane lamellar and in-plane $\pi$-stack scattering is still evident.

a

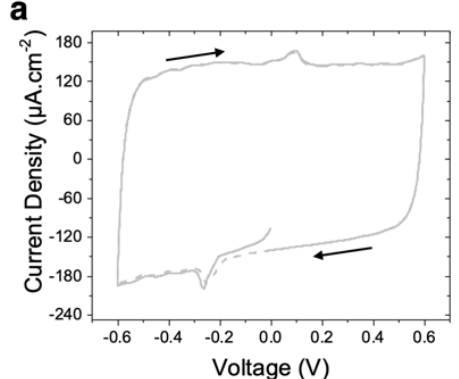

b

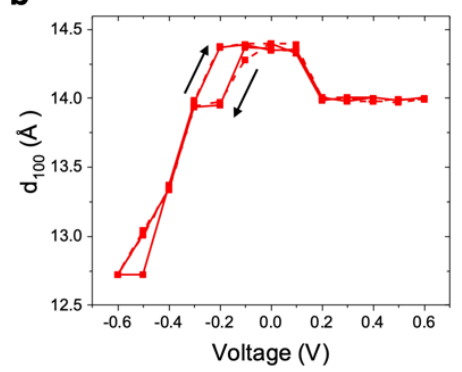

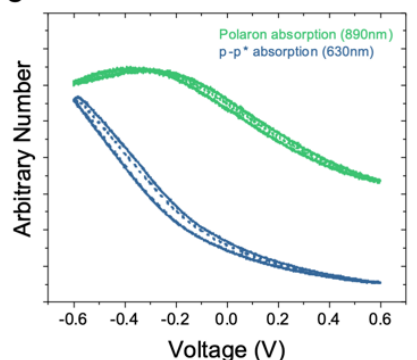

Figure S2. (a) Cyclic voltammogram during operando GIWAXS at a sweep rate of $5 \mathrm{mV} / \mathrm{s}$. (b) Lamellar d-spacing and (c) neutral and polaron absorptions during cyclic voltammetry. Two cycles plotted, first cycle solid lines, second cycle dashed lines. Note the d-spacing hysteresis in (b) between -0.2 and $-0.3 \mathrm{~V} \mathrm{vs} \mathrm{Ag} / \mathrm{AgCl}$.

a

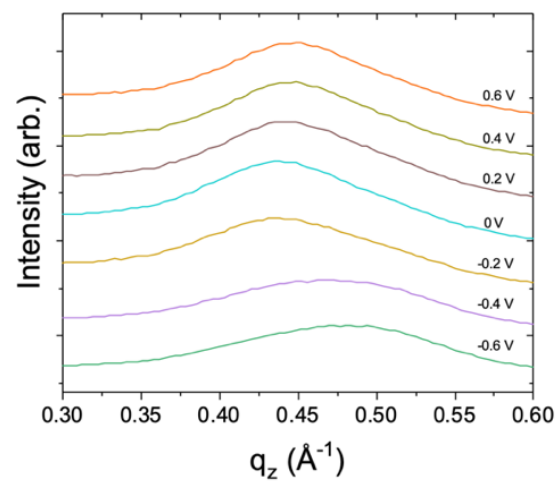

b

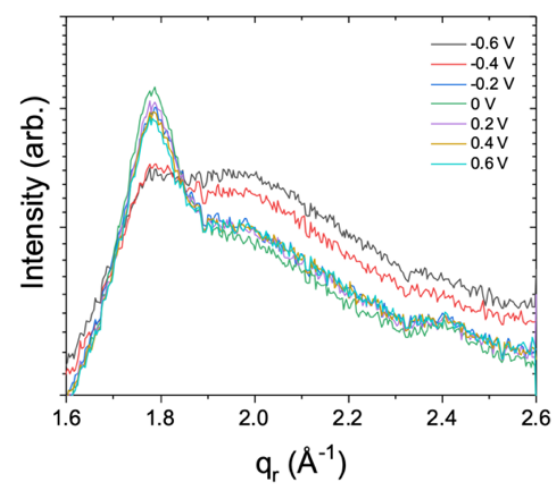

Figure S3. Line cuts of (a) out-of-plane lamellar and (b) in-plane $\pi$-stack scattering peaks collected from equilibrated fixed potential GIWAXS scattering plots. Line cuts in (a) have been offset to accentuate the peak shift. 
Transient and Kinetic Model Fitting. Due to the large time step (0.5s) over which scattering was averaged, the d-spacing transient was fit empirically with an asymmetric sigmoidal function.

$d(t)=\frac{d_{f}-d_{i}}{\left(1+\exp \left(\frac{t-t_{i}}{\tau_{d}}\right)\right)^{5}}+d_{i}$

The exponent was fixed large and positive, while the time constant $\left(\tau_{d}\right)$ and inflection point $\left(t_{i}\right)$ were allowed to vary, and $d_{i}$ and $d_{f}$ were the initial and final lamellar $d$-spacings.

The normalized chronocoulometric transients were fit with a simple exponential function.

$\frac{q(t)}{q_{f}}=-\exp \left(\frac{-t}{\tau_{q}}\right)+1$

Where $q_{f}$ is the final equilibrated charge and $\tau_{q}$ is the $R C$ charging time constant.

The transients of the $\pi-\pi^{*}$ absorption $\left(A_{n}\right)$ during dedoping ( 0 to $-0.6 \mathrm{~V}$ vs $\mathrm{Ag} / \mathrm{AgCl}$ ) were fit as the final product of two consecutive elementary reactions.

$A_{n}(t)=\left(A_{n, f}-A_{n, i}\right)\left(1+\frac{k_{b p \rightarrow p}}{k_{p \rightarrow n}-k_{b p \rightarrow p}} \exp \left(-k_{p \rightarrow n} t\right)-\frac{k_{p \rightarrow n}}{k_{p \rightarrow n}-k_{b p \rightarrow p}} \exp \left(-k_{b p \rightarrow p} t\right)\right)+A_{n, i}$

Where $A_{n, i}$ and $A_{n, f}$ were the initial and final $\pi-\pi^{*}$ absorption values, respectively, and $k_{b p \rightarrow p}$ and $k_{p \rightarrow n}$ were the bipolaron to polaron and polaron to neutral rate constants, respectively. For all other potential steps the $\pi-\pi^{*}$ absorption transients were fit as a single elementary reaction.

$A_{n}(t)=\left(A_{n, i}-A_{n, f}\right) \exp \left(-k_{x} t\right)+A_{n, f}$

Where $k_{x}$ represented $k_{n \rightarrow p}$ and $k_{p \rightarrow n}$ depending of potential step. The transients of the polaron absorption $\left(A_{p}\right)$ during doping and dedoping between 0 and $-0.6 \mathrm{~V} v \mathrm{Ag} / \mathrm{AgCl}$ were fit as the intermediary of two consecutive elementary reactions. During doping taking the form:

$A_{p}(t)=N\left(\frac{k_{n \rightarrow p}}{k_{p \rightarrow b p}-k_{n \rightarrow p}}\left((1+\alpha) \exp \left(-k_{n \rightarrow p} t\right)-(1-\alpha) \exp \left(-k_{p \rightarrow b p} t\right)\right)\right)+A_{p, f}$

And during dedoping taking the form:

$A_{p}(t)=B P\left(\frac{k_{b p \rightarrow p}}{k_{p \rightarrow n}-k_{b p \rightarrow p}}\left((1+\alpha) \exp \left(-k_{b p \rightarrow p} t\right)-(1-\alpha) \exp \left(-k_{p \rightarrow n} t\right)\right)\right)+A_{p, f}$ 
Where $\alpha$ was an empirical fit parameter, and $N$ and $B P$ are constants proportional to the initial neutral and bipolaron populations, respectively; and $k_{n \rightarrow p}, k_{p \rightarrow b p}, k_{b p \rightarrow p}$, and $k_{p \rightarrow n}$ were the neutral to polaron, polaron to biopolar, bipolaron to polaron, and polaron to neutral rate constants, respectively. For all other potential steps the polaron absorption transients were fit as a single elementary reaction.

$A_{p}(t)=\left(A_{p, i}-A_{p, f}\right) \exp \left(-k_{x} t\right)+A_{p, f}$

Where $k_{x}$ represented $k_{p \rightarrow n}$ and $k_{p \rightarrow b p}$ depending of potential step, and $A_{p, i}$ was the initial polaron absorption value. 